\section{Smyelin for glia}

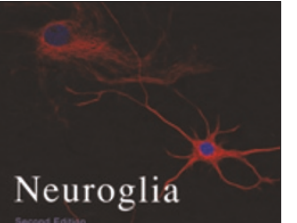

Neuroglia, 2nd edition

Edited by Helmut Kettenmann and Bruce R Ransom

Oxford University Press, 2004

624 pp., hardcover, $\$ 149.50$

ISBN 0195152220

\section{Reviewed by Ben A Barres}

"The brain is a world consisting of a number of unexplored continents and great stretches of unknown territory."

- Santiago Ramon y Cajal

I like to study glia for the same reason that I like to play the viola. Most folks study neurons or play the violin, so glial biologists and violists are always in short supply. But as a violist, one rarely gets to play a solo; the same is true for glia. Perhaps that's why I very much like this new edition of Neuroglia. It is a wonderful book focused entirely on glial cells.

About $90 \%$ of cells in the human brain are glial cells, yet their functions remain mysterious. Oligodendrocytes myelinate, but nearly half the glial cells in the brain are astrocytes, long thought to be passive support cells. Yet each of these beautiful little cells contacts blood vessels and ensheathes several thousand synapses, where they are positioned to powerfully control the function of every circuit in our brain. Any injury of the brain produces a widespread alteration in astrocyte phenotype called reactive gliosis, whose function is also mysterious but likely to be pivotal to the pathophysiology of the injured CNS. Therefore, understanding the role of CNS glia is one of the most important territories in all of neuroscience today.

Fortunately, there has been an acceleration of glial research in the ten years since the first edition of Neuroglia. Neuroscience textbooks tend to neglect glia, so this new edition is without doubt the best place to turn to for some glial education. The book has been greatly shortened-a good thing - and is divided into three main sections, on glia properties, functions and roles in disease. The first section focuses on history, morphology, development, physiology and biochemistry. I particularly enjoyed the first chapter, with its historical perspective on the origin of the concept of neuroglia. Other excellent chapters summarize glial development and the recent findings that some cells in the adult brain previously thought to be glia are in fact multipotent neural stem cells. Alas, some investigators prefer to keep referring to these stem cells as astrocytes or glial cells. Should every cell in the brain that is not a neuron be called a glial cell? I vote no.

The second section focuses on functional interactions of glial cells with neurons. Glia myelinate axons, have immune functions, help induce and maintain the blood-brain barrier, regulate ion and neurotransmitter concentrations, serve trophic roles and help guide axons. The chapters on myelination are excellent and remind us how many mysteries remain in regard to even this best-known role of glia. We do not know the identities of the main axonal signals that induce (or inhibit) myelin formation, and we do not understand the mechanism of myelin wrapping itself. Next, several excellent chapters summarize the explosion of new evidence that astrocytes may actively help to control the structure, function and plasticity of synapses. One quibble, however: although astrocytes in culture can release glutamate, there are many reasons to think that this does not occur normally within the brain. Here I would urge caution.

In reading these chapters, one realizes that most of the suggested roles for astrocytes remain hypothetical. We really don't understand how the blood-brain barrier is formed, or what role astrocytes play in this process. Similarly, much has been made of the role of astrocytes in controlling neuronal survival, but do astrocytes in vivo actually help neurons to survive? I know of no evidence one way or the other. Additional chapters teach us that there are several other glial cell classes in the brain including microglia and a class of oligodendrocyte precursor cells also called synantocytes, whose functions in a normal brain are entirely unknown. Perhaps my favorite chapter in the second part of the book is the one on the central role of astrocytes in neuroenergetics. This and several other chapters reveal how very different neurons and glia are in their metabolic pathways, and how far we have to go in understanding the significance of these differences to both normal and abnormal brain function. I predict that by the time of the book's third edition, we are finally going to see some major steps forward in understanding glial function-and I would bet that many of these steps forward are going to come from invertebrate genetic model systems such as Caenorhabiditis elegans (yes, there are glia in worms) and Drosophila melanogaster.

If there are any deficiencies in the first two sections of the book, they reflect the experimental limitations of doing glial research. Many studies of glia have been largely descriptive rather than question-oriented. Fortunately, it is now increasingly possible to do more hypothesisdriven research about glia. Finally, the chapters in the third section of the book focus on the role of glia in various neurological diseases. We are still in the Stone Age, here; if we don't understand the normal function of glia, then how can we understand their abnormal function? These chapters beautifully illustrate how important a better basic understanding of glial cells is likely to be for developing treatments for such neurological conditions as multiple sclerosis, stroke and Alzheimer disease.

All in all, Kettenmann and Ransom have once again done a great job of putting together this terrific book, a book which is just one of their many important contributions to the community on behalf of the neuroglia. I forgot to mention another reason why glia and violas remind me of each other: they are both the butt of jokes. What do you call a glial cell when it is happy? Smyelin! 